\title{
Intraoperative Findings and Outcome of Latarjet Procedure
}

\section{Andreas M. Riederer1, Thomas Lattmann², Christoph Meier ${ }^{3}$, Heike A. Bischoff-Ferrari ${ }^{4}$, Michael Dietrich1, Patrick Grueninger' ${ }^{1}$}

\author{
${ }^{1}$ Division of Traumatology/Orthopedics, Department of Surgery, Waid City Hospital, Zurich, Switzerland \\ ${ }^{2}$ Division of Vascular Surgery, Department of Surgery, Cantonal Hospital Winterthur, Winterthur, Switzerland \\ ${ }^{3}$ Division of Traumatology/Orthopedics, Department of Surgery, Cantonal Hospital Winterthur, Winterthur, Switzerland \\ ${ }^{4}$ Department of Geriatrics and Aging Research, University Hospital Zurich and University of Zurich, Zurich, Switzerland \\ Email: Andreas.Riederer@gmx.de
}

How to cite this paper: Riederer, A.M., Lattmann, T., Meier, C., Bischoff-Ferrari, H.A., Dietrich, M. and Grueninger, P. (2018) Intraoperative Findings and Outcome of Latarjet Procedure. Open Journal of Orthopedics, 8, 273-289.

https://doi.org/10.4236/ojo.2018.87031

Received: May 23, 2018

Accepted: July 10, 2018

Published: July 13, 2018

Copyright (C) 2018 by authors and Scientific Research Publishing Inc. This work is licensed under the Creative Commons Attribution International License (CC BY 4.0)

http://creativecommons.org/licenses/by/4.0/

\begin{abstract}
Background: The purpose of this case series was to retrospectively compare radiological, clinical and functional outcomes and complications of diagnostic arthroscopy with open Latarjet procedures pre- and postoperatively within one year after surgery. Additionally we compared the pathologic findings during diagnostic arthroscopy with the radiological findings in preoperative contrast enhanced CT or MRI scans. Methods: Between 07/2009 and 11/2013 46 cases with unidirectional antero-inferior shoulder instability were enrolled, 4 cases were lost during the follow-up within one year postoperatively. Plain radiographs, contrast enhanced multislice studies, Instability Severity Index, Constant, Duplay and Rowe Scores were obtained preoperatively. Diagnostic arthroscopy was performed in all cases prior to open Latarjet procedure. At one year follow-up Constant, Duplay and Rowe Scores were obtained; position and consolidation of the coracoid transfer were assessed by conventional $\mathrm{x}$-ray studies. Results: At one year follow-up a significant improvement of all scores was recorded (Constant Score 95.8 vs. 86.7; Duplay Score 93.7 vs. 25.2, Rowe Score 98.1 vs. 31.7, $(p<0.001))$. During arthroscopy 28 of 42 HillSachs-lesions were considered as engaging. 8 HAGL (humeral avulsions of the gleno-humeral ligaments) and 4 IGHL (inferior gleno-humeral ligaments) lesions were detected of which none were suggested during the preoperative radiological investigation. Complications observed were: screw migration (5), hematoma (1), infection (1) and recurrence (1). Conclusion: The Latarjet procedure is a reliable technique with very good clinical outcomes. Diagnostic arthroscopy is a valuable tool to detect HAGL- and IGHL-lesions and to visualize the engaging potential of Hill-Sachs-lesions. An additional arthroscopy may help to indicate a Latarjet procedure and to address concomitant pathologies. Level of evidence: Level IV, case series.
\end{abstract}




\section{Keywords}

Shoulder Instability, Shoulder Arthroscopy, Latarjet Procedure, HAGL Lesion, Bankart Lesion, Instability Severity Index Score, Engaging Hill-Sachs Lesion

\section{Introduction}

Shoulder stabilization surgery for antero-inferior shoulder instability has advanced in the last three decades with further developments in arthroscopy methods and suture anchors to address shoulder instability [1] [2] [3]. Different shoulder stabilization techniques can be categorized in anatomical or nonanatomical reconstruction, soft tissue or bony reconstruction and open or arthroscopic approaches. One common option consists of soft-tissue repair using open or arthroscopic capsulolabral repair (Bankart-repair) with the aim to reconstruct native anatomy to ensure sufficient stability of the shoulder, thus relying on soft tissue quality and sufficient anterior glenoid bone stock [4] [5] [6]. However unsatisfactory outcomes have been described regarding the Bankartrepair in presence of miscellaneous pathologies associated with shoulder instability, including humeral avulsions of the gleno-humeral ligaments (HAGL-lesion) [7] [8], lesions of the inferior gleno-humeral ligaments (IGHL-lesion), bony glenoid defects/osseous Bankart-lesions and engaging Hill-Sachs-lesions [2] [9]. In these aforementioned situations or previous failed Bankart-repair the Latarjet procedure, the transfer of the coracoid with the attached conjoined tendon to the anterior glenoid rim, has been described as useful alternative technique for anterior shoulder stabilization [1] [10] [11] [12]. Notably, also the Latarjet procedure improved since its first description in 1954 [13]. The stability of the shoulder after Latarjet procedure is supported by several factors. The transferred coracoid graft reconstructs the anterior glenoid rim in bone deficient cases and prevents an engaging of a Hill-Sachs-lesion at the antero-inferior glenoid rim through an increased articular glenoid surface [2] [3] [14]. In addition the also transferred conjoined tendon acts in combination with the lowered part of the subscapularis-split as a dynamic reinforcement of the antero-inferior joint section in abduction and external rotation. The Latarjet-procedure biomechanically supports both ligamentous and osseous insufficiencies and is therefore indicated for treatment of both conditions [15]. The development of the Instability Severity Index Score (ISI-Score) [16] aimed at selection of patients with anterior shoulder instability who would benefit from surgery. Furthermore, the use of multi-slice radiological investigations offers a 3 dimensional view and detailed structural information to help select patients who will benefit from surgery. However, despite technical improvements certain pathologies such as HAGL-lesions, IGHLlesions or the significance of an engaging Hill-Sachs-lesion may be underestimated in CT and MRI investigations, as these are static and not dynamic inves- 
tigations. The main goal of this case series was to compare radiological, clinical and functional outcomes and complications of diagnostic arthroscopy followed by open Latarjet procedures pre- and postoperatively. Additionally we compared pathological findings during diagnostic arthroscopy with the radiological findings in preoperative contrast enhanced CT or MRI scans. The primary endpoint of this study was the final follow-up one year postoperative. We hypothesized that at final follow-up the clinical global function (Constant Score) as well as the subjective shoulder stability (Duplay and Rowe Score) should improve. Our second hypothesis was that despite extensive preoperative investigations the extent of injury accountable for the resulting antero-inferior shoulder instability is underestimated and diagnostic arthroscopy helps in decision finding for Latarjet procedure and to address concomitant pathologies.

\section{Material and Methods}

\subsection{Patients}

Between July 2009 and November 2013 diagnostic shoulder arthroscopy with subsequent open Latarjet procedure was performed on 45 patients with antero-inferior instability in 46 shoulders at the Department of Surgery in the Waid City Hospital in Zurich. Forty-one patients with 42 affected shoulders with a minimum follow-up of 11 months were included in this case series. Excluded were 4 of the initial 45 patients that were lost to follow-up at 3 months postoperatively. These 4 patients had "perfect" results regarding postoperative Constant, Duplay and Rowe Scores as well as "perfect" radiological follow-up 3 months postoperatively but wished to conclude therapy at this time and leave the study.

The local ethics committee approved this study (Req-2017-00412). All patients signed informed consents for the operative treatment, possible supplement surgery in case of unanticipated pathologies or conversion to an appropriate technique referring to the intraoperatively found pathologies and all follow-up investigations. Clinical examinations and plain radiological studies were evaluated and compared pre-operatively and at follow-up. Patients receiving other stabilization procedures were not included in this study.

\subsection{Operative Procedure}

All procedures were performed with patients under general anesthesia with an interscalene catheter for postoperative pain control (Perifix catheter, B. Braun Medical AG, Switzerland, Accufuser Silicone Baloon Infuser-Continuos Type, Woo Young Medical Co, LTD, France/Korea, Ropivacain 0.2\% continuous, Ropivacain $0.6 \%$ boli). The patient was placed in a standardized beach-chair position, arm traction of 2 to $3 \mathrm{~kg}$ depending on the patient's build was applied during diagnostic shoulder arthroscopy. Cefuroxime as antibiotic prophylaxis was routinely administered perioperative in patients without previous shoulder surgery. In revision surgery procedures tissue samples regarding low grad infections were obtained before administration of cefuroxime. The gleno-humeral joint 
was infiltrated with $20 \mathrm{ml}$ of diluted epinephrine ( $1 \mathrm{ml}$ of epinephrine $(1 \mathrm{mg} / \mathrm{ml})$ and $19 \mathrm{ml}$ of normal saline solution) to decrease intraoperative bleeding. A standardized survey of the glenohumeral joint documented typical instability associated pathologies, in particular bony pathologies such as Hill-Sachs-, bony Bankart-lesions and defects of the glenoid or inverted pear formation. In addition the engaging potential of Hill-Sachs-lesions was visualized by positioning the arm in $90^{\circ}$ abduction and maximal external rotation. A positive engaging was recorded when the Hill-Sachs-lesion cavity fell in the glenoid without any additional force [2]. Secondary intraarticular pathologies requiring mandatory surgery were addressed arthroscopically in the same session. For tenodesis of the long head of the biceps tendon (LHBT) or reconstruction of an isolated subscapularis lesion the technique described by Grueninger et al. was used [17]. After complete survey of the gleno-humeral joint an open Latarjet procedure was performed with at least one of the following pathologies present: Engaging HillSachs-lesion, HAGL-, IGHL-lesion, bony Bankart-lesion/glenoid bone deficiency $(>25 \%$ of anterior-posterior glenoid diameter or $<25 \%$ of anterior-posterior glenoid diameter plus other above named lesion), or revision surgery after failed Bankart-repair. The Latarjet procedure was performed as a standardized adapted mini-open procedure [18] using the "Bristow Latarjet Instability Shoulder System" of DePuy Synthes Mitek Sports Medicine as described by Lafosse et al. 2007, who developed and is using this system in an arthroscopic technique [3]. In particular, attention is given to place the coracoid graft flush with the glenoid surface and to place the implanted screws at least $7 \mathrm{~mm}$ from the glenoid margin to result in the desired graft placement and to avoid lateral overhang of the graft [19]. All operations were performed by senior surgeons, 33 of 42 operations by the senior author, 9 by the third author.

\subsection{Rehabilitation}

All patients were immobilized postoperatively in a sling for 7 days. On the first postoperative day physiotherapy was initiated with active hand and elbow movement and passive shoulder pendulum without strain. From the $2^{\text {nd }}$ until the $6^{\text {th }}$ postoperative week shoulder motion was increased to active mode without immobilization with restricted external rotation at $0^{\circ}$ with elbow at the side. At 7 weeks postoperatively pain adapted weight bearing, free external rotation and active motion were allowed, patients also started recreational activities like swimming, jogging and cycling. At 3 months postoperatively patients regained full weight bearing. Patients who reached full range of motion could begin with contact sports, over-head sports, martial arts and sking.

\subsection{Clinical and Radiological Evaluation}

All patient's medical history of dislocations, beginning of shoulder instability and the Instability Severity Index Score (ISI-Score) [16] were recorded preoperatively. Either the operating surgeon or the first author performed a standardized 
clinical examination preoperatively and during final follow-up including the Constant, Duplay and Rowe Score [6] [20] [21]. Pre- and postoperative all patients underwent a plain radiographic evaluation including antero-posterior view in neutral rotation, Neer projection and Bernageau view [22]. Preoperative contrast enhanced CT scans in 34 cases and contrast enhanced MRI scans in 8 cases were obtained of the affected shoulder. The position and consolidation of the coracoid grafts were assessed by conventional radiograph studies using antero-posterior view in neutral rotation, Neer projection and Bernageau view at final follow-up 1 year postoperative, measurement of coracoid placement was accomplished by the first author. Development of osteoarthritis was measured according to the Samilson/Prieto classification [23].

\subsection{Statistical Analysis}

Statistical analysis in this study was performed using the paired $t$ test under supervision of an epidemiologist at the Centre of Aging and Mobility at the University of Zurich. All results are presented as means with standard deviations, with ranges in parentheses. A double-sided $P<0.05$ was considered statistically significant.

\section{Results}

\subsection{Preoperative Evaluation and Intraoperative Findings}

Between July 2009 and November 2013 diagnostic shoulder arthroscopy with subsequent open Latarjet procedure was performed on 45 patients with antero-inferior instability in 46 shoulders. Of these 45 patients 4 were lost to follow-up and therefore excluded. Of the remaining 41 patients there were 42 shoulders with antero-inferior instability. Of those 5 were female and 36 patients were male. The mean age was $30.3 \pm 9.9$ (range 17 - 52 years). Affected were 14 right and 28 left shoulders, one patient had bilateral antero-inferior shoulder instability (Table 1). On average the patients reported 3.1 (range 1 - 10) dislocations in their history and 10 patients received previous surgery (7 Bankart-repairs, 2 with Re-Bankart-repairs, 1 with Re-Bankart-repair and EdenHybinette).

Table 1. Study population.

\begin{tabular}{cc}
\hline No patients/gender/cases & 41 patients/36 male, 5 female/42 cases \\
\hline Affected Side & 14 right/28 left/1 bilaterally \\
Age & $30.3 \pm 9.9$ years (range $17-52$ years) \\
Quantity of dislocations & 3.1 (range $1-10)$ \\
Number of cases with previous surgery & 10 \\
Type of previous surgery & $7 \times$ Bankart-repair, $2 \times 2$ Bankart-repair, $1 \times 2$ \\
\end{tabular}

Values are given as numbers with standard deviation and range in parentheses. 
Preoperatively Constant, Duplay, Rowe and ISI-Score were obtained. The determined preoperative scores were: Constant Score $86.7 \pm 14.2$ (range 22 - 100), Duplay Score $25.2 \pm 15.3$ (range -20 - 50) and Rowe Score $31.7 \pm 10.3$ (range 5 55 ). The mean ISI-Score was $5.8 \pm 1.8$ (range 1 - 10), 6 (14.3\%) patients were under 20 years old, 24 (57.1\%) patients were athletes in competitive sports, 38 $(90.5 \%)$ patients took part in contact sports or in sports with forced overhead movements, 19 (45.2\%) patients had a positive Gagey-Test [24]. Fifteen (35.7\%) patients had an ISI-Score of greater than 6, indicating preoperatively a Latarjet procedure. The dynamic arthroscopic evaluation of the 42 shoulders showed the following pathologies: During arthroscopy all of the 42 radiological diagnosed Hill-Sachs-lesions could be confirmed. 28 of these 42 (66.7\%) Hill-Sachs-lesions were considered as engaging Hill-Sachs-lesions. The direct visual examination with the arthroscope confirmed that the Hill-Sachs-lesion was located parallel to the anterior glenoid and the Hill-Sachs-lesion engaged the corner of the glenoid in a functional position e.g. abduction and external rotation as mentioned above [2]. Additional 8 HAGL (19.0\%) and 4 IGHL (9.5\%) lesions were detected from which none were suggested in the preoperative radiologic investigation. In addition $11(26.2 \%)$ bony Bankart-lesions or chronic glenoid bone deficiencies were visualized, one with a glenoid bone deficiency of $25 \%$, the remaining 10 cases had a glenoid bone deficiency of $10 \%-15 \%$ and at least one other pathology indicating a Latarjet procedure (engaging Hill-Sachs-lesion, HAGL-, IGHL-lesion or revision surgery after failed Bankart-repair). In one case (2.4\%) a reversed Bankart-lesion not diagnosed during the preoperative evaluation was surprisingly detected. This lesion was simultaneously treated arthroscopically with posterior labral repair using suture anchors and showed uneventfully for the further course. During arthroscopy 6 (14.3\%) SLAP II lesions were identified and treated with tenodesis of the long head of the biceps tendon with suture anchors, 1 (2.4\%) SLAP lesion was repaired arthroscopically with suture anchors. Only 4 of these 7 SLAP-lesions were found during the preoperative evaluation. Intraoperative $2(4.8 \%)$ isolated Lafosse type II subscapularis-lesions were found, of which one was detected in the preoperative evaluation. Both were repaired arthroscopically [25]. After diagnostic arthroscopy an open Latarjet procedure was performed with at least one of the following pathologies present: Engaging Hill-Sachs-lesion, HAGL-, IGHL-lesion, bony Bankart-lesion/glenoid bone deficiency ( $>25 \%$ of anterior-posterior glenoid diameter or $<25 \%$ of anterior-posterior glenoid diameter plus other above named lesion), or revision surgery (Table 2). Patients receiving revision surgery showed in 6 out of $10(60 \%)$ cases a low-grade infection with Propionibacterium acnes and were treated with a double antibiotic therapy of Rifampicin plus Ciprofloxacin or Levofloxacin or Clindamycin orally for 3 months.

\subsection{Clinical and Radiological Outcome}

The mean follow-up time for the 42 cases in this series was 14.6 months \pm 6.4 
Table 2. Found pathology.

\begin{tabular}{cccc}
\hline Pathology & $\begin{array}{c}\text { Suggested in } \\
\text { CT/MRI }\end{array}$ & $\begin{array}{c}\text { Arthroscopic } \\
\text { Visualization }\end{array}$ & $\begin{array}{c}\text { Supplementary } \\
\text { Surgery }\end{array}$ \\
\hline $\begin{array}{c}\text { Hill-Sachs-lesion } \\
\text { Engaging }\end{array}$ & 42 & 42 & - \\
Hill-Sachs-lesion & - & 28 & - \\
HAGL-lesion & 0 & 8 & - \\
IGHL-lesion & 0 & 4 & $\begin{array}{c}6 \times \text { arthroscopic tenodesis of LHBT } \\
\text { for SLAP II, } 1 \times \text { SLAP Repair } \\
\text { SLAP }\end{array}$ \\
Rotator-Cuff-lesion & 1 & 7 & $2 \times$ arthroscopic double row repair of \\
\end{tabular}

Found pathology according to CT/MRI, under arthroscopic direct visualization and supplementary surgery, Abbreviation: LHBT, Long Head of Biceps Tendon.

months (range 11 - 43 months). At follow-up a significant $(p<0.001)$ improvement of all scores was recorded (Table 3 and Figure 1). The Constant Score improved from preoperatively $86.7 \pm 14.2$ (range $22-100$ ) to postoperatively $95.8 \pm$ 4.4 (range 79 - 100), Duplay Score improved from preoperatively $25.2 \pm 15.3$ (range $-20-50)$ to postoperatively $93.7 \pm 9.4$ (range $55-100$ ). Likewise Rowe Score increased from preoperatively $31.7 \pm 10.3$ (range 5 - 55) to $98.1 \pm 3.1$ (range 90 - 100) postoperatively. Table 4 shows the different scores categorized as excellent, good, fair and poor (excellent: 91 - 100 points, good: 76 - 90 points, fair: $51-75$ points and poor: $<51$ points). Revision surgery was performed in three cases (7.1\%). In one (2.4\%) case, a 23 years old male patient had to undergo revision surgery 3 weeks postoperative due to a postoperative hematoma. One patient $(2.4 \%)$, a 17 years old male, acquired a tattoo in the surgical incision area two weeks after surgery likely resulting in a purulent infection with Propionibacterium acnes. After revision surgery the infection was treated successfully with Rifampicin plus Clindamycin orally for 3 months. One (2.4\%) antero-superior recurrence was recorded in the patient in our series with bilateral antero-inferior shoulder instability. The 52 years old patient had to undergo revision surgery on his right shoulder and was treated with arthroscopic remplissage of the $M$. infraspinatus and open anatomic glenoid restoration using a J-shaped iliac crest bone graft.

At follow-up one year postoperatively position and consolidation of the coracoid graft was assessed by conventional x-ray studies using antero-posterior view in neutral rotation, Neer projection and Bernageau view. In 5 (11.9\%) cases a partial loosening of screws without clinical significance (no symptoms, no recurrence, no subluxation, no local irritation) was observed. One total (2.4\%) and two partial lyses (4.8\%) of the coracoid were observed in these 5 cases, all of them without clinical impact. Other cases of coracoid lyses were not found. Measurement of the graft position in ap and Bernageau position showed 2 (4.8\%) grafts not in a $2-5$ o'clock position but in a $1-4$ o'clock position (for a 
Table 3. Scores preoperatively and postoperatively.

\begin{tabular}{ccccc}
\hline & ISI-Score & Constant Score & Duplay Score & Rowe Score \\
\hline \multirow{2}{*}{ Preoperatively } & $5.83 \pm 1.78$ & $86.7 \pm 14.2$ & $25.2 \pm 15.3$ & $31.7 \pm 10.3$ \\
& $(1-10)$ & $(22-100)$ & $(-20-50)$ & $(5-55)$ \\
Postoperatively & - & $95.8 \pm 4.4$ & $93.7 \pm 9.4$ & $98.1 \pm 3.1$ \\
& & $(79-100)$ & $(55-100)$ & $(90-100)$ \\
$p$-value & - & $<0.001$ & $<0.001$ & $<0.001$ \\
\hline
\end{tabular}

Values are given as mean with standard deviation and range in parentheses.

Table 4. Score results.

\begin{tabular}{ccccc}
\hline & Excellent & Good & Fair & Poor \\
\hline Constant Score & $39(92.9 \%)$ & $3(7.1 \%)$ & 0 & 0 \\
Duplay Score & $24(57.1 \%)$ & $16(38.1 \%)$ & $2(4.8 \%)$ & 0 \\
Rowe Score & $39(92.9 \%)$ & $3(7.1 \%)$ & 0 & 0 \\
\hline
\end{tabular}

Score results 1 year postoperatively graded into excellent (100 - 91 points), good (90 - 76 points), fair (75 51 points), poor $(<51$ points) given as number of cases with percentage in parentheses.

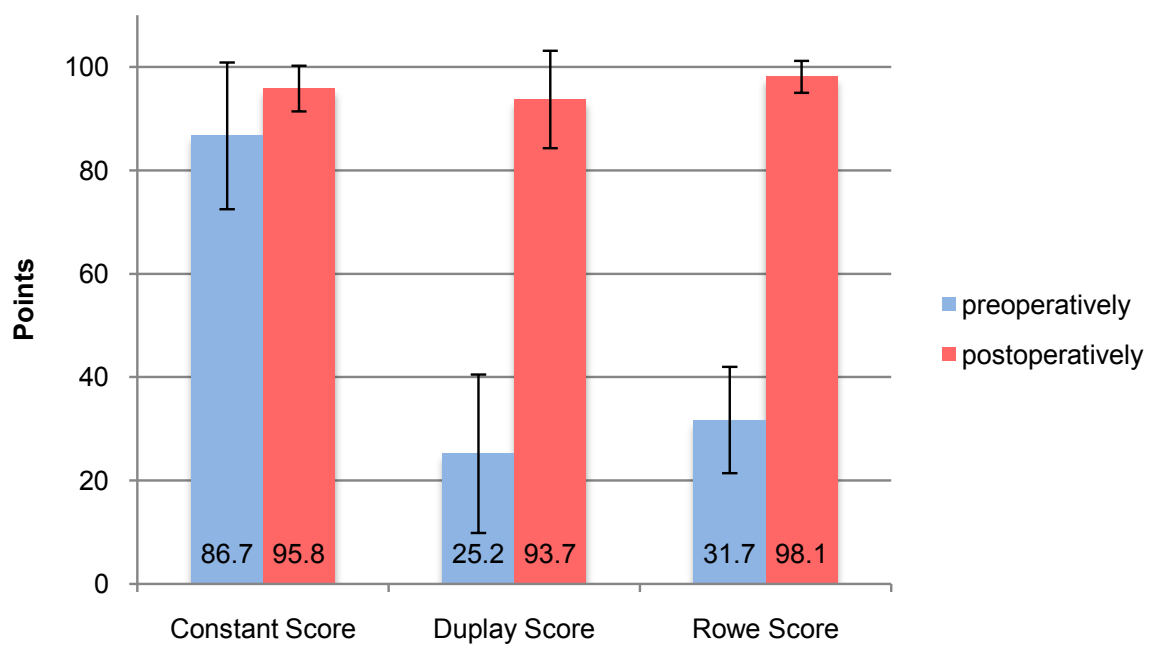

Figure 1. Scores preoperatively and postoperatively.

right shoulder). Furthermore 4 (9.5\%) of the coracoid grafts were located 2 $4 \mathrm{~mm}$ medial of the anterior glenoid rim rather than being positioned flush with the glenoid surface which was detected in 38 cases (90.5\%). None of the 42 coracoid grafts were positioned laterally of the glenoid rim (Figure 2).

Additionally we investigated for signs of instability arthropathy according to the Samilson classification [23]. In our series one case (2.4\%), with preexisiting chronic nervus axillary neuropraxy and plexus brachialis injury after spine surgery and multiple shoulder dislocations, was graded as mild osteoarthritis Samilson $I^{\circ}$. At follow-up this case progressed to a severe shoulder osteoarthritis Samilson $\mathrm{III}^{\circ}$ evidently due to the excentric position of the humeral head (Figure $3 \mathrm{E} / \mathrm{F}$ ). 


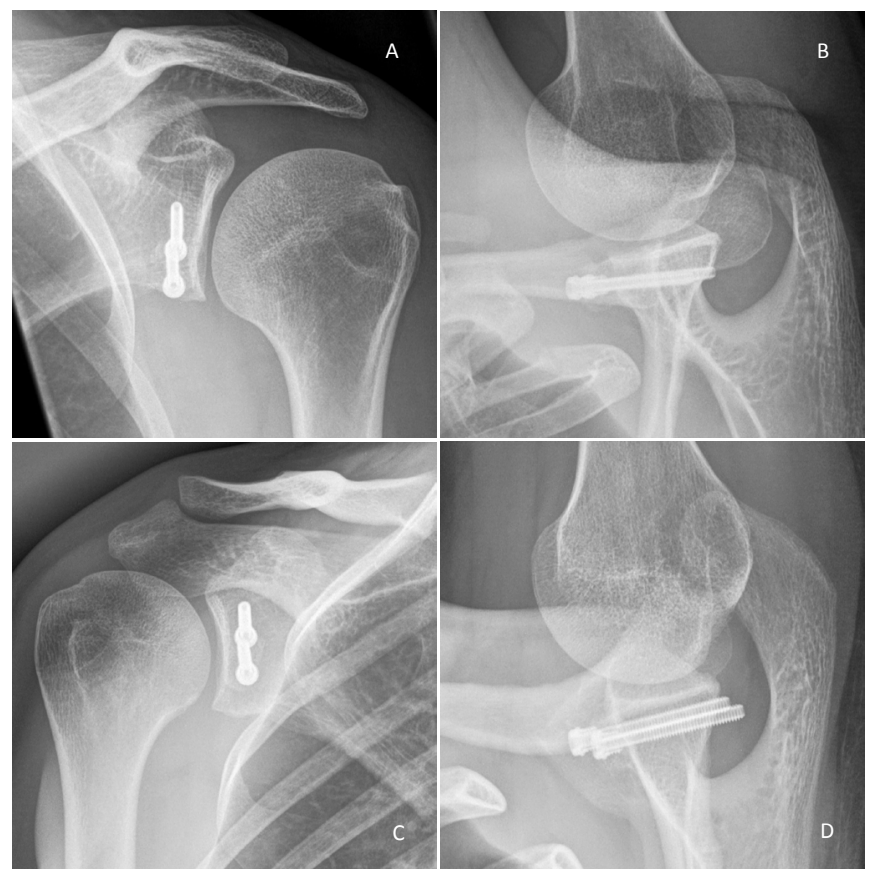

Figure 2. Examples of postoperative radiographs. Postoperative radiographs of coracoid graft position at final follow-up. A: Antero-posterior view of the left shoulder of a 28 -year male patient, coracoid graft in perfect position; B: Bernageau view of the left shoulder of a 28-year male patient, coracoid graft in perfect position; C: Antero-posterior view of the right shoulder of a 17-year old female patient, coracoid graft in high position; D: Bernageau view of the left shoulder of a 22 -year old male patient, coracoid graft in medial position.

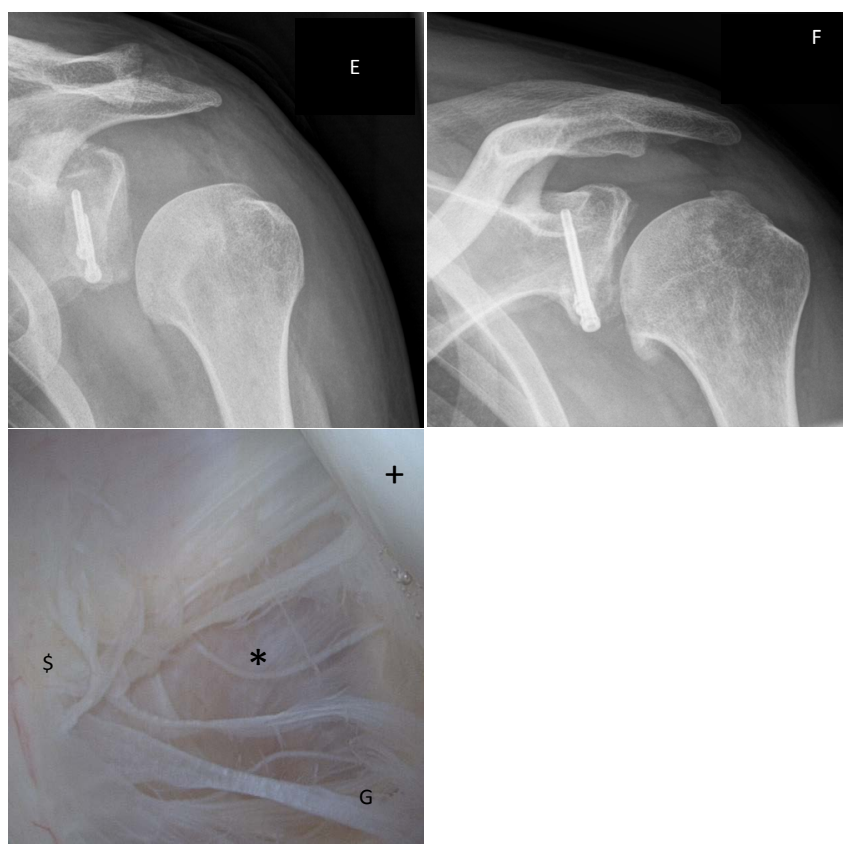

Figure 3. Example of osteoarthritis. E and F: Antero-posterior view of a patient with excentric humeral head position due to pre-existing N. axillaris and plexus brachialis injury developing osteoarthritis; E: 1st day postoperative; F: 9 months postoperative; G: Intraoperative visualization of HAGL-lesion; +: Humeral head, *: HAGL-lesion, \$: IGHL. 


\section{Discussion}

\subsection{Preoperative Evaluation and Intraoperative Findings}

According to the preoperatively determined ISI-Score only 15 patients had an ISI-Score greater than 6 (ISI-Score maximum 10) where an open Latarjet procedure would be indicated [16]. One disadvantage of the ISI-Score is that it accentuates young athletes in competitive sports, as both circumstances (Age under 20 and competitive sports) contribute to the ISI-Score with 2 points each. Regarding the ISI-Score a Latarjet procedure is therefore not indicated when the patient's age exceeds 20 years and the patient is not participating in competitive sports. In our study 6 (14.3\%) patients were under 20 years old and 24 (57.1\%) patients were athletes in competitive sports. Furthermore preoperative radiological multislice examination with contrast enhanced CT or MRI studies suggested none of the 8 HAGL-, none of the 4 IGHL-lesions and none of the 28 engaging Hill-Sachs-lesions. As already mentioned in the introduction, the significance of an engaging Hill-Sachs-lesion may be underestimated in CT and MRI investigations, as these are static and not (like during arthroscopy) dynamic investigations. Additional rotator cuff lesions during antero-inferior shoulder dislocation are age depending and occur mainly in patients older than 40 years [26] [27] [28] [29]. Therefore in patients with an age above 40 years contrast enhanced MRIs were obtained preoperatively to specify potential additional rotator cuff lesions. Patients with an age under 40 years were evaluated preoperatively with contrast enhanced CTs.

Arthroscopy is the diagnostic reference standard for the detection of HAGL-lesions (example of intraoperative visualization of HAGL-lesion in Figure 3 G) [8] [30] [31]. Three large clinical series describe the prevalence of HAGL-lesions in MRI scans ranging from 2\% (6 of 307 patients) to $7.5 \%$ (41 of 547 patients) to $9.4 \%$ ( 6 of 64 patients) [8] [32] [33]. However a sensitivity of 0.50 for the MRI diagnosis is only mentioned in one study [33]. One recent study reports a prevalence of HAGL-lesions of $4.64 \%$ (9 of 194 patients) with a sensitivity of MRI in detecting HAGL-lesions of 0.44 and a specificity of 0.97 [31]. In our series 4 of the 8 cases with HAGL-lesions received a preoperative MRI, the other 4 received a preoperative $\mathrm{CT}$ according to the study protocol listed in Methods (Age $<40=\mathrm{CT}$, Age $>40=\mathrm{MRI}$ ). Therefore it is possible that we could have diagnosed more HAGL-lesions preoperatively if all patients would have received a preoperative MRI. Of the cases with previous stabilization surgery only 4 were not included in the aforementioned patients with an ISI-Score above 6 and radiological indication for a Latarjet procedure. Relating to the preoperative evaluation of ISI-Score, previous stabilization surgery and radiological suggestion of HAGL- or IGHL-lesions or engaging Hill-Sachslesions or glenoid bone deficiencies, 21 cases (50\%) showed a proper indication for a Latarjet procedure. The value of diagnostic arthroscopy could be seen in our case series as we found pathologies during arthroscopy not diagnosed in our preoperative evaluation with multislice studies and therefore strengthening the 
indication for a Latarjet procedure in all cases. According to literature these indications consist of existence of at least one of the following pathologies: Previous surgery, engaging Hill-Sachs-lesion, HAGL- or IGHL-lesion or glenoid bone deficiency, where a Bankart-repair would have lead to inferior results [1] [10] [11] [12]. The particular advantage of diagnostic arthroscopy is the dynamic exanimation unlike the static setting of CT or MRI studies offering a direct visualization and assessment of the underlying pathology. Moreover supplement surgeries for 7 SLAP-lesions, 2 subscapularis-lesions and 1 reversed Bankart-lesion were performed, only 4 of the 7 SLAP-lesions, resp. 1 of the subscapularis-lesions were suggested during the preoperative radiological workup. Therefore 5 of the 42 patients (11.9\%) would most likely have unsatisfactory outcomes if these pathologies would not have been detected and addressed during the arthroscopy. Arrigoni et al. [34] published a case series of 33 patients who underwent diagnostic arthroscopy before open Latarjet procedure. In this case series $55 \%$ of the patients had previous surgery, $73 \%$ showed associated intra-articular lesions (64\% sustained a type II SLAP lesion) and 64\% had engaging Hill-Sachs-lesions. Arrigoni's study supports our results of a high incident of engaging Hill-Sachs-lesions and demonstrates the value of arthroscopy to address simultaneous shoulder lesions. Our study strengthens not only the already described usefulness of arthroscopy to address these concomitant lesions but may also contribute aid in decision finding for the proper stabilization procedure as above mentioned lesions like HAGL- or IGHL-lesions are often overlooked when not particularly seeked for [7] [8]. Our intraoperative diagnostic findings also support the findings of a biomechanical cadaver study of the IGHL published by Bigliani et al. [35] in 1991, where lesions of the IGHL were detected in $40 \%$ at the glenoid insertion, $35 \%$ in the ligament midsubstance and $25 \%$ at the humeral insertions site.

\subsection{Clinical and Radiological Outcome in Comparison to Literature}

At follow-up the collected Constant, Duplay and Rowe Scores all showed a significant $(p<0.001)$ improvement. Unfortunately, a direct comparison to other studies is limited due to the heterogenic follow-up and the use of different scoring methods (Table 5). Burkhart et al. [36] reported in 2007 a case series of 102 Latarjet procedure of whom 47 patients were available for postoperative examination at a mean follow-up of 59 months. In Burkhart's study the patients underwent diagnostic arthroscopy to quantify glenoid bone loss and to identify and address concomitant pathologies arthroscopically. In case of a glenoid bone loss of $>25 \%$ or a concomitant engaging Hill-Sachs-lesion, Burkhart proceeded to an open Latarjet procedure as these were considered indications for a Latarjet Procedure. At follow-up a mean Constant Score of 94.4 and a mean Duplay Score of 91.7 could be shown. Complications noted were 2 hematomas and a recurrence rate of $4 \%$. Radiological signs of 2 screw loosenings and 1 fibrous union were described. Schmid et al. [11] published in 2012 results of a case series of 49 cases 
Table 5. Comparison of studies reporting outcomes of Latarjet procedures.

\begin{tabular}{|c|c|c|c|c|c|c|c|c|c|}
\hline & $\begin{array}{l}\text { No of } \\
\text { Cases }\end{array}$ & $\begin{array}{l}\text { Follow-up } \\
\text { (Months) }\end{array}$ & Recurrence & Subluxation & $\begin{array}{l}\text { Constant } \\
\text { Score }\end{array}$ & $\begin{array}{l}\text { Duplay } \\
\text { Score }\end{array}$ & Rowe Score & Complications & $\begin{array}{c}\text { Coracoid } \\
\text { Graft/Implant }\end{array}$ \\
\hline $\begin{array}{c}\text { Allain et } \\
\text { al. } 1998\end{array}$ & 58 & $\begin{array}{c}171 \\
(120-276)\end{array}$ & 0 & $1(2 \%)$ & 84 & - & - & $\begin{array}{c}\text { Total } 5(9 \%) \\
3(5 \%) \\
\text { Infection } \\
1(2 \%) \\
\text { Frozen shoulder } \\
1(2 \%) \\
\text { Humeral fracture }\end{array}$ & $\begin{array}{c}2(3 \%) \\
\text { Non-union } \\
10(17 \%) \text { Partial } \\
\text { lysis }\end{array}$ \\
\hline $\begin{array}{l}\text { Schmid } \\
\text { et al. } \\
2012\end{array}$ & $\begin{array}{c}49(41 \\
\text { clinical/radiological, } \\
8 \text { telephone survey) }\end{array}$ & $\begin{array}{c}38 \\
(23-63)\end{array}$ & 0 & $2(4 \%)$ & $\begin{array}{c}84.6 \\
(40-100)\end{array}$ & - & - & $\begin{array}{c}\text { Total } 6(12 \%) \\
4(8 \%) \\
\text { Delayed wound } \\
\text { healing } \\
2(4 \%) \\
\text { Frozen shoulder }\end{array}$ & $1(2 \%)$ Non-union \\
\hline $\begin{array}{c}\text { Burkhart } \\
\text { et al. } \\
2007\end{array}$ & $\begin{array}{c}102 \\
\text { (47 clinical, } 55 \\
\text { telephone survey) }\end{array}$ & $\begin{array}{l}59 \pm 18.5 \\
(32-108)\end{array}$ & $4(4 \%)$ & 0 & $\begin{array}{c}94.4 \\
(82-100)\end{array}$ & $\begin{array}{c}91.7 \\
(75-100)\end{array}$ & - & $\begin{array}{c}\text { Total } 6(6 \%) \\
2(2 \%) \\
\text { Hematomas } \\
4(4 \%) \\
\text { Recurrence }\end{array}$ & $\begin{array}{c}2(2 \%) \\
\text { Screw loosening } \\
1(1 \%) \\
\text { Fibrous union }\end{array}$ \\
\hline $\begin{array}{l}\text { Shah et } \\
\text { al. } 2012\end{array}$ & $\begin{array}{c}48 \\
\text { (45 follow-up, } 3 \text { lost) }\end{array}$ & $\begin{array}{c}9.4 \\
(6-55)\end{array}$ & $4(8 \%)$ & 0 & - & - & - & $\begin{array}{c}\text { Total } 12(25 \%) \\
3(6 \%) \\
\text { Infections } \\
4(8 \%) \\
\text { Recurrence } \\
5(10 \%) \\
\text { Nerve damage }\end{array}$ & $\begin{array}{c}8(18 \%) \\
\text { Non-union }\end{array}$ \\
\hline $\begin{array}{l}\text { Riederer } \\
\text { et al. } \\
2018\end{array}$ & 42 & $\begin{array}{c}14.6 \pm 6.4 \\
(11-43)\end{array}$ & $1(2.4 \%)$ & 0 & $\begin{array}{l}95.8 \pm 4.4 \\
(79-100)\end{array}$ & $\begin{array}{l}93.7 \pm 9.4 \\
(55-100)\end{array}$ & $\begin{array}{l}98.1 \pm 3.1 \\
(90-100)\end{array}$ & $\begin{array}{c}\text { Total } 3(7.1 \%) \\
1(2.4 \%) \\
\text { Hematoma } \\
1(2.4 \%) \\
\text { Infection } \\
1(2.4 \%) \\
\text { Recurrence }\end{array}$ & $\begin{array}{c}1(2.4 \%) \\
\text { Total lysis } \\
2(4.8 \%) \text { Partial } \\
\text { lysis of coracoid } \\
\text { graft } \\
5(11.9 \%) \text { Screw } \\
\text { loosening }\end{array}$ \\
\hline
\end{tabular}

Data with standard deviation and range in parentheses where available.

for open Latarjet procedure without concurrent diagnostic arthroscopy after previous shoulder stabilization of whom 41 cases were available for postoperative examination with a mean follow-up of 38 months (the remaining 8 were contacted by telephone survey) showing a mean Constant Score of 84.6. Schmid considered recurrence shoulder instability, glenoid bone loss of at least one-third of the antero-posterior glenoid diameter and absence of fatty infiltration of the subscapularis muscle greater than Goutallier stage II as indications for an open Latarjet procedure. Schmid reported no recurrence, a subluxation rate of $4 \%$ and a total complication rate of $12 \%$ consisting of 4 delayed wound healing, 2 frozen shoulders and 1 malunion with pain. Schmid described the position of the coracoid graft as flush in $73 \%$, medial in $15 \%$, lateral in $12 \%$. Likewise osteoarthritis in 11 patients (27\%), of which 9 had already preoperative osteoarthritis, was de- 
scribed. Shah et al. [37] reported a case series of 48 cases (of whom 45 were available for follow-up) receiving an open Latarjet procedure with a mean follow-up of 9.4 months with a recurrence rate of $8 \%$ in 2012. Shah considered glenoid bone loss $>30 \%$ and unsuccessful arthroscopic or open capsule and labral repair as indications for open Latarjet procedure. According to Shah diagnostic arthroscopy prior to the Latarjet procedure was performed in 18 of these 48 cases $(37.5 \%)$ to assess soft tissue quality and bone involvement that had not been appreciated on preoperative imaging in patients having undergone unsuccessful arthroscopic Bankart-repair. Shah described a rather high total complication rate of $25 \%$ with $6 \%$ infection, $8 \%$ recurrence and $10 \%$ neurological complications. Furthermore Shah reported non-union of the coracoid graft in 8 (18\%) cases. Long term results of 58 shoulders after open Latarjet procedure without prior diagnostic shoulder arthroscopy with a mean follow-up of 14.3 years (171 months) were published by Allain et al. [38] in 1998. No recurrence, a subluxation rate of $2 \%$ and a total complication rate of $9 \%$ consisting of 3 infections, 1 frozen shoulder and 1 humeral fracture were mentioned. Allain et al. also reported the position of the coracoid graft: the results were graded as perfect ( 0 - $5 \mathrm{~mm}$ medial of the anterior glenoid rim) in $41 \%$, lateral of the anterior glenoid rim in $53 \%$ and more than $5 \mathrm{~mm}$ medial of the glenoid rim in $5 \%$. Allain also reported non-union of the coracoid graft in 3\% and partial lysis in 17\%. Assigning the grading of the coracoid position according to Allain, we found all coracoid grafts in our case series in a perfect position, as we report 38 (90.5\%) of the transferred coracoid being flush with the glenoid rim, 4 (9.5\%) of the coracoid grafts were located medial of the anterior glenoid rim (range $2-4 \mathrm{~mm}$ ) and none of the 42 coracoid grafts in our study were positioned laterally of the glenoid rim. According to Allain et al. the correct position of the coracoid graft is the most important factor in the development of postoperative glenohumeral osteoarthritis, the other 2 factors identified are untreated rotator cuff lesions and intra- or postoperative complications like infection or recurrence. Table 5 shows the results of our study in comparison to these aforementioned studies. In our series especially no neurological complications were noted in the postoperative course and follow-up. One patient suffered from pre-existing brachial plexus and axillary nerve damage due to cervical spine injury and cervical disc prolapse without association with the performed shoulder surgery. Allain et al. showed that a high rate of lysis of the coracoid graft is not related to a high recurrence rate as he reported 10 cases of partial coracoid lysis and no recurrence in his long-term study. Our results support that finding as the radiological detected lysis of the coracoid had no influence on clinical outcome in our series, which also supports that the sling effect of the conjoint tendon crossing the subscapularis seems to have a significant effect on the stability of the shoulder in external rotation in $90^{\circ}$ abduction. We, as Allain and Burkhart as well, did not augment the anterior capsule with the stump of the coraco-acromion ligament [36] [38]. Shah and Schmid used the coraco-acromion ligament to augment the anterior capsule 
as described by others [37] [39]. The Constant Score mobility subscore in our series showed no restriction in external rotation.

\subsection{Strength and Limitations}

Compared to the aforementioned studies [11] [36] [37] [38] our case series includes similar quantity of controlled patients and indicates comparable results relating to recurrence and subluxation rate. Moreover we report significant improvements of the 3 measured scores (Constant, Duplay, Rowe) with a low complication rate. One limitation of our study is the short follow-up compared to the studies of Burkhart [36], Schmid [11] and Allain [38]. Possible development of osteoarthritis despite perfect placement of the coracoid graft is described by Allain et al. and is not regularly monitored beyond our follow-up. Therefore the later development of osteoarthritis cannot be ruled out despite our promising short-term results. In the compared studies the majority of recurrence occurred within 12 months, but also recurrences later on in follow-up have been reported [11] [36] [37] [38] Potential higher recurrence rates are therefore possible in long-term results.

\section{Conclusion}

Progress in arthroscopic skills lead to technical notes and reports of arthroscopic Latarjet-procedure that allowed an all-arthroscopic approach in treatment of anterior shoulder instability [3] [14] [40]. However despite high number of cases these reports lack long-term results. Moreover the arthroscopic approach to the Latarjet procedure and to this complex pathology can be demanding and complications due to the proximity of the axillar and musculocutaneous nerve and the brachial plexus to the coracoid can be severe and hazardous. Therefore the arthroscopic Latarjet procedure will be reserved for all but the most experienced and able arthroscopic surgeons in high volume centers. Another reason to still perform mini open Latarjet procedure is to teach young colleagues in open shoulder stabilizing surgery. This would not be possible in a low or medium volume center for the arthroscopic Latarjet procedure. The Latarjet procedure is a reliable technique with very good clinical outcomes and no neurological complication occurred in our series. Of interest lysis of the coracoid had no influence on clinical outcome in our series. Diagnostic arthroscopy is a valuable tool to detect HAGL and IGHL lesions and to visualize the engaging potential of Hill-Sachs-lesions. An additional arthroscopy may help to indicate a Latarjet procedure, despite missing indications in multislice examinations or in ISI-Score.

\section{References}

[1] Boileau, P., et al. (2006) Risk Factors for Recurrence of Shoulder Instability after Arthroscopic Bankart Repair. The Journal of Bone and Joint Surgery. American Volume, 88, 1755-1763. https://doi.org/10.2106/JBJS.E.00817

[2] Burkhart, S.S. and De Beer, J.F. (2000) Traumatic Glenohumeral Bone Defects and 
Their Relationship to Failure of Arthroscopic Bankart Repairs: Significance of the Inverted-Pear Glenoid and the Humeral Engaging Hill-Sachs Lesion. Arthroscopy, 16, 677-694. https://doi.org/10.1053/jars.2000.17715

[3] Lafosse, L., et al. (2007) The Arthroscopic Latarjet Procedure for the Treatment of Anterior Shoulder Instability. Arthroscopy, 23, 1242 e1-5.

[4] Mohtadi, N.G., et al. (2005) Arthroscopic versus Open Repair for Traumatic Anterior Shoulder Instability: A Meta-Analysis. Arthroscopy, 21, 652-658. https://doi.org/10.1016/j.arthro.2005.02.021

[5] Owens, B.D., et al. (2011) Surgical Trends in Bankart Repair: An Analysis of Data from the American Board of Orthopaedic Surgery Certification Examination. The American Journal of Sports Medicine, 39, 1865-1869.

https://doi.org/10.1177/0363546511406869

[6] Rowe, C.R., Patel, D. and Southmayd, W.W. (1978) The Bankart Procedure: A Long-Term End-Result Study. The Journal of Bone and Joint Surgery. American Volume, 60, 1-16. https://doi.org/10.2106/00004623-197860010-00001

[7] Bach, B.R., Warren, R.F. and Fronek, J. (1988) Disruption of the Lateral Capsule of the Shoulder. A Cause of Recurrent Dislocation. The Journal of Bone and Joint Surgery. British Volume, 70, 274-276. https://doi.org/10.1302/0301-620X.70B2.3346302

[8] Wolf, E.M., Cheng, J.C. and Dickson, K. (1995) Humeral Avulsion of Glenohumeral Ligaments as a Cause of Anterior Shoulder Instability. Arthroscopy, 11, 600-607. https://doi.org/10.1016/0749-8063(95)90139-6

[9] Walch, G., et al. (1995) Arthroscopic Stabilization for Recurrent Anterior Shoulder Dislocation: Results of 59 Cases. Arthroscopy, 11, 173-179. https://doi.org/10.1016/0749-8063(95)90063-2

[10] Bhatia, S., et al. (2014) The Outcomes and Surgical Techniques of the Latarjet Procedure. Arthroscopy, 30, 227-235. https://doi.org/10.1016/j.arthro.2013.10.013

[11] Schmid, S.L., et al. (2012) The Latarjet Procedure for the Treatment of Recurrence of Anterior Instability of the Shoulder after Operative Repair: A Retrospective Case Series of Forty-Nine Consecutive Patients. The Journal of Bone and Joint Surgery. American Volume, 94, e75. https://doi.org/10.2106/JBJS.K.00380

[12] Yamamoto, N., et al. (2010) Stabilizing Mechanism in Bone-Grafting of a Large Glenoid Defect. The Journal of Bone and Joint Surgery. American Volume, 92, 2059-2066. https://doi.org/10.2106/JBJS.I.00261

[13] Latarjet, M. (1954) Treatment of Recurrent Dislocation of the Shoulder. Lyon Chirurgical, 49, 994-997.

[14] Rosso, C., et al. (2016) Technical Guide and Tips on the All-Arthroscopic Latarjet Procedure. Knee Surgery, Sports Traumatology, Arthroscopy, 24, 564-572. https://doi.org/10.1007/s00167-014-3038-x

[15] Schulze-Borges, J., et al. (2013) Biomechanical Comparison of Open and Arthroscopic Latarjet Procedures. Arthroscopy, 29, 630-637. https://doi.org/10.1016/j.arthro.2012.12.003

[16] Balg, F. and Boileau, P. (2007) The Instability Severity Index Score. A Simple Pre-Operative Score to Select Patients for Arthroscopic or Open Shoulder Stabilisation. The Journal of Bone and Joint Surgery. British Volume, 89, 1470-1477. https://doi.org/10.1302/0301-620X.89B11.18962

[17] Grueninger, P., et al. (2014) Arthroscopic Repair of Traumatic Isolated Subscapularis Tendon Lesions (Lafosse Type III or IV): A Prospective Magnetic Resonance 
Imaging-Controlled Case Series with 1 Year of Follow-Up. Arthroscopy, 30, 665-672. https://doi.org/10.1016/j.arthro.2014.02.030

[18] Mercier, N. and Saragaglia, D. (2011) Mini-Open Latarjet Procedure for Recurrent Anterior Shoulder Instability. Advances in Orthopedics, 2011, Article ID: 656205.

[19] Young, A.A., et al. (2013) Coracoid Graft Dimensions after Harvesting for the Open Latarjet Procedure. Journal of Shoulder and Elbow Surgery, 22, 485-488. https://doi.org/10.1016/j.jse.2012.05.036

[20] Constant, C.R. and Murley, A.H. (1987) A Clinical Method of Functional Assessment of the Shoulder. Clinical Orthopaedics and Related Research, 214, 160-164. https://doi.org/10.1097/00003086-198701000-00023

[21] Walch, G. (1991) La luxation récidivante antérieure d'épaule, table ronde SO.F.C.OT. Revue de Chirurgie Orthopedique et Reparatrice de L'appareil Moteur, 77, 177-191.

[22] Bernageau, J., et al. (1976) Value of the Glenoid Profil in Recurrent Luxations of the Shoulder. Revue de Chirurgie Orthopedique et Reparatrice de L'appareil Moteur, 62, 142-147.

[23] Samilson, R.L. and Prieto, V. (1983) Dislocation Arthropathy of the Shoulder. The Journal of Bone and Joint Surgery. American Volume, 65, 456-460. https://doi.org/10.2106/00004623-198365040-00005

[24] Gagey, O.J. and Gagey, N. (2001) The Hyperabduction Test. The Journal of Bone and Joint Surgery. British Volume, 83, 69-74.

https://doi.org/10.1302/0301-620X.83B1.10628

[25] Lafosse, L., et al. (2007) Structural Integrity and Clinical Outcomes after Arthroscopic Repair of Isolated Subscapularis Tears. The Journal of Bone and Joint Surgery. American Volume, 89, 1184-1193. https://doi.org/10.2106/00004623-200706000-00005

[26] Atef, A., et al. (2016) Prevalence of Associated Injuries after Anterior Shoulder Dislocation: A Prospective Study. International Orthopaedics, 40, 519-524. https://doi.org/10.1007/s00264-015-2862-z

[27] Gumina, S., Carbone, S. and Postacchini, F. (2009) Occult Fractures of the Greater Tuberosity of the Humerus. International Orthopaedics, 33, 171-174. https://doi.org/10.1007/s00264-007-0512-9

[28] Pevny, T., Hunter, R.E. and Freeman, J.R. (1998) Primary Traumatic Anterior Shoulder Dislocation in Patients 40 Years of Age and Older. Arthroscopy, 14, 289-294. https://doi.org/10.1016/S0749-8063(98)70145-8

[29] Sonnabend, D.H. (1994) Treatment of Primary Anterior Shoulder Dislocation in Patients Older than 40 Years of Age. Conservative versus Operative. Clinical Orthopaedics and Related Research, 304, 74-77.

[30] Acid, S., et al. (2012) Preoperative Imaging of Anterior Shoulder Instability: Diagnostic Effectiveness of MDCT Arthrography and Comparison with MR Arthrography and Arthroscopy. American Journal of Roentgenology, 198, 661-667. https://doi.org/10.2214/AJR.11.7251

[31] Saqib, R., Funk, L. and Harris, J. (2016) Humeral Avulsion of Glenohumeral Ligaments-Detection on Magnetic Resonance Arthrography. Journal of Arthroscopy and Joint Surgery, 3, 3-6. https://doi.org/10.1016/j.jajs.2016.02.001

[32] Bokor, D.J., Conboy, V.B. and Olson, C. (1999) Anterior Instability of the Glenohumeral Joint with Humeral Avulsion of the Glenohumeral Ligament. A Review of 41 Cases. The Journal of Bone and Joint Surgery. British Volume, 81, 93-96. 
https://doi.org/10.1302/0301-620X.81B1.9111

[33] Bui-Mansfield, L.T., et al. (2002) Humeral Avulsions of the Glenohumeral Ligament: Imaging Features and a Review of the Literature. American Journal of Roentgenology, 179, 649-655. https://doi.org/10.2214/ajr.179.3.1790649

[34] Arrigoni, P., et al. (2008) The Value of Arthroscopy before an Open Modified Latarjet Reconstruction. Arthroscopy, 24, 514-519. https://doi.org/10.1016/j.arthro.2007.11.021

[35] Bigliani, L.U., et al. (1992) Tensile Properties of the Inferior Glenohumeral Ligament. Journal of Orthopaedic Research, 10, 187-197. https://doi.org/10.1002/jor.1100100205

[36] Burkhart, S.S., et al. (2007) Results of Modified Latarjet Reconstruction in Patients with Anteroinferior Instability and Significant Bone Loss. Arthroscopy, 23, 1033-1041. https://doi.org/10.1016/j.arthro.2007.08.009

[37] Shah, A.A., et al. (2012) Short-Term Complications of the Latarjet Procedure. The Journal of Bone and Joint Surgery. American Volume, 94, 495-501.

https://doi.org/10.2106/JBJS.J.01830

[38] Allain, J., Goutallier, D. and Glorion, C. (1998) Long-Term Results of the Latarjet Procedure for the Treatment of Anterior Instability of the Shoulder. The Journal of Bone and Joint Surgery. American Volume, 80, 841-852. https://doi.org/10.2106/00004623-199806000-00008

[39] Bradley Edwards, T. and Walch, G. (2002) The Latarjet Procedure for Recurrent Anterior Shoulder Instability: Rationale and Technique. Operative Techniques in Sports Medicine, 10, 25-32. https://doi.org/10.1053/otsm.2002.28776

[40] Boileau, P., Mercier, N. and Old, J. (2010) Arthroscopic Bankart-Bristow-Latarjet (2B3) Procedure: How to Do It and Tricks to Make It Easier and Safe. Orthopedic Clinics of North America, 41, 381-392. https://doi.org/10.1016/j.ocl.2010.03.005 